\title{
Comparison of Analytical and Experimental Force in Cylindrical Workpiece Drawing Process
}

\author{
O. Fanidia,*, A.A. Kostryukov ${ }^{b}$, A.V. Shchedrinc, I.Yu. Ignatkinc \\ a Ecole Nationale Supérieure d'Arts et Métiers, Hassan II University, Casablanca, Morocco, \\ ${ }^{b}$ Machine-Building Plant, Demikhovsky, Orekhovo-Zuevo, Russia, \\ ${ }^{c}$ MT13, Engineering technology, Bauman Moscow State Technical University, Moscow, Russia.
}

Keywords:

Drawing force

Micro-relief

Deformation zone

Lubricants

* Corresponding author:

Omar Fanidi

E-mail: omarfanidi75@gmail.com

Received: 6 November 2020

Revised: 3 January 2021

Accepted: 24 February 2021

\begin{abstract}
A B S T R A C T
A mathematical module for predicting and analyzing the drawing force is formed and verified. The process used in the current study is drawing. Geometric parameters of the deformation zone and the "selective transfer phenomenon" are also taken into consideration. The experimental verification is conducted through two tests: In the first test, the experimental blanks are cylindrical rods (steel 45, length $150 \mathrm{~mm}$ ), and are preliminary machined by turning, and the drawplate made of steel 9xc, are used. Regular micro-relief (RMR) hardens the working surfaces of the drawplate. In the second test, the same conditions are applied except for the samples, which represent solid cylindrical billets made of steel 45 with a complicatedly modified surface layer, including a brass film, regular microgeometry, and a "servo-witte" copper film. As a result, in the first experiment, the discrepancies are in the range of $-29.1 \%$ up to $+23 \%$. In the second experiment, the discrepancies are in the range of $+1.38 \%$ up to $+48.9 \%$.
\end{abstract}

(C) 2021 Published by Faculty of Engineering

\section{INTRODUCTION}

Drawing is a highly efficient method for machining, finishing, and hardening holes on workpieces with various dimensions, structures and shapes [1-3].

Applied to solid cylindrical profiles, the current methods of processing are systemically and intensively improved through the use of selforganizing tribotechnologies, which include regularizing microgeometry and surface modification of a drawing tool and workpiece, as well as various methods of influence by metal-clad lubricants that allow realizing the fundamental scientific principle "Garkunov-Kragelsky effect, friction without wear" [4-15].

This machining method allows to reduce the drawing force by $25-75 \%$, and to increase quality and productivity up to two times. It also allows forming a protective film ("servo-Witte") which contributes to a significant reduction in the cost of drawing tools [9-15].

When choosing technological equipment for the implementation of the relevant drawing operations, it is necessary to accurately predict the processing forces [1,2,17-21]. At the same time, the 
most accurate theoretical model for predicting and analyzing the drawing forces of solid cylindrical billet is Vorontsov's expression [19], which additionally has a wide physical visibility. In this work, the mathematical module for predicting the drawing force of continuous cylindrical workpieces was developed and was verified. In this elaboration, the parameters of the deformation zone and the "selective transfer phenomenon" were taken into account. For this reason two experimental studies are applied to two different samples.

\section{ANALYTICAL MODEL}

Vorontsov relied on bronze, brass, and copper [19] as basic elements in his experiments dedicated to the verification of the theoretical model of the drawing force expression. Another particularity in his experiments is that he focused on the impact which the conical part angle of the deformation element has on the drawing force. The optimal angle is in the interval $5-9^{\circ}$. However, the model proposed by Vorontsov does not take into account the actual geometry of the deformation zone $\Delta R_{b}$, the properties of metalclad lubricants, the phenomenon of "selective transfer under friction" in the form of a "servo film" $h_{s w}[22,23]$, nor the roughness parameters $\chi_{b}, R_{s w}$, and $H_{b \max }$. To eliminate these imperfections and to enrich this study, the experimental and theoretical results obtained previously are used to formulate the mathematical model (1) of the drawing force.

$$
\left\{\begin{array}{c}
q_{d}^{\mathrm{T}}=0.25\left(D_{l}-2 h_{s w}\right) \bar{\sigma}_{s}\left[\left(2+f \frac{\sin \alpha}{1-\cos \alpha}\right) \ln \left(\frac{D_{b} \pm 2 \Delta R_{b}+2 h_{s w}}{D_{l}-2 h_{s w}}\right)+\frac{1-\cos \alpha}{\sin \alpha}+2 f \frac{L_{l}}{\left(D_{l}-2 h_{s w}\right)}\right] ; \\
F_{d}^{\mathrm{T}}=\pi q_{d}^{\mathrm{T}}\left(D_{l}-2 h_{s w}\right) ; \\
f=f_{a}^{\mathrm{T}}=\frac{E\left(\chi_{b}+1\right)}{\pi K_{1} K_{2}\left(\chi_{b}+1.5\right)} \sqrt{\frac{\varepsilon_{b} H_{b \max }}{K_{3} R_{s w}}} ; \\
E\left(\frac{\pi K_{1} K_{2}\left(\chi_{b}+1.5\right)}{E\left(\chi_{b}+1\right)} \tau_{0} \sqrt{\frac{K_{3} R_{s w}}{\varepsilon_{b} H_{b \max }}}+\beta\right)+\left(0.28 \sqrt{\frac{\varepsilon_{b} H_{b \max }}{R_{s w}}}\right) .
\end{array}\right.
$$

Where:

$q_{d}^{\mathrm{T}}$ - Theoretical specific (linear) drawing force, $\mathrm{N} / \mathrm{mm}$;

$D_{l}$ - Diameter of the working channel of the deforming drawplate in a calibrating ribbon, $\mathrm{mm}$;

$h_{s w}$ - The thickness of the part of the modified layer in the form of a protective film ("servo-witte"), mm;

$\bar{\sigma}_{s}$ - The average yield stress of the material processed according to (in) the deformation zone, MPa;

$f$ - Sliding friction coefficient;

$f_{a}$ and $f_{d}$ - Respectively, the adhesive and the deformation component of the coefficient of sliding friction;

$\alpha$ - The angle of the working cone of the deforming drawplate;

$D_{b}$ - Diameter of the workpiece before drawing, taking into account the thickness of the additional layer as a result of regularization of the surface microgeometry and the application of an anti-friction coating [11, $12,15], \mathrm{mm}$;
$\Delta R_{b}$ - Parameter of the deformation zone in the form of a sign and wave height of non-contact deformation arising on the working cone of the drawplate, mm; (fig. 3, fig. 4 and fig. 5);

$L_{l}$ - Width of the calibrating ribbon deforming the drawplate, $\mathrm{mm}$;

$F_{d}^{\mathrm{T}}$ - Theoretical total drawing force, $\mathrm{N}$;

$q_{a}^{\mathrm{T}}$ - Theoretical actual contact pressure on the working cone of the deforming drawplate, $\mathrm{MPa}$;

$E$ - Modulus of elasticity, MPa;

$\chi_{b}$ - Parameter of the law of the altitudinal distribution of micro-speaks of the rough surface of the workpiece before drawing;

$K_{1}, K_{2}$ and $K_{3}$-Coefficients of the general solution of the Hertz-Belyaev contact problem [23];

$R_{s w}$ - The radius of curvature at the summit of a single micro protrusion of the rough surface of the working channel of the deforming drawplate, $\mu \mathrm{m}$;

$\varepsilon_{b}$ - Relative deformation of the maximum micro protrusion of the rough surface of the workpiece as a result of drawing; 
$H_{b \max }$ - The height of the maximum micro protrusion of the rough surface of the workpiece for drawing, $\mu \mathrm{m}$;

$\tau_{0}$ - Strength adhesive connections at $q_{a}^{\mathrm{T}}=$ $0 \mathrm{MPa}$, [23];

$\beta$ - Piezocoefficient influence $q_{a}^{\mathrm{T}}$ on strength adhesive connections.

Hence, the derivation and determination of the above parameters and coefficients are partially considered in $[5,19,23]$.

\section{EXPERIMENTAL STUDY}

\subsection{First experimental study}

For the first experimental base, for testing the theoretical model of the drawing force (1), the process of drawing solid cylindrical billets made of steel 45 (174-187 HB) through a deforming drawplate made of steel 9XC (58-61 HRC) is considered [10]. In this process, the tool used is similar to that of the works $[8,9,13$, 14]; their working surface is strengthened by the creation of a regular micro-relief in the form of single-turn threaded channels with a radius of $1.5 \mathrm{~mm}$, a pitch of $0.5 \mathrm{~mm}$, and an initial depth in the calibration-strip section of $10 \mu \mathrm{m}$. Tests were carried out on five specimens that have the following diameters: $D_{b}=$ $20.091 \mathrm{~mm} ; D_{b}=20.206 \mathrm{~mm} ; D_{b}=20.295 \mathrm{~mm}$; $D_{b}=20.389 \mathrm{~mm} ; D_{b}=20.489 \mathrm{~mm}$. The actual absolute deformation is calculated by the expression $i_{a}=D_{b}-D_{l}$; where $D_{l}=20 \mathrm{~mm}$ is a diameter over the calibration bland (the diameter tool). Therefore the first specimen is deformed by the actual absolute deformation $i_{a}=0.091 \mathrm{~mm}$, the second by $i_{a}=0.206 \mathrm{~mm}$, the third by $i_{a}=0.295 \mathrm{~mm}$, the fourth by $i_{a}=0.389 \mathrm{~mm}$, and the fifth by $i_{a}=0.489 \mathrm{~mm}$. The length of the part that undergoes drawing is $150 \mathrm{~mm}[10,13]$. Profilometers Maud 201 and Maud 296 are used to obtain the profile-grams. The longitudinal profile-grams of the workpieces before drawing (after turning) are shown in Figure 1, where: $R a_{b}$, - the surface roughness parameter of the sample blanks before drawing. The figure 1 is used to determine the parameters $\chi_{b}$ and $H_{b \text { max }}$.

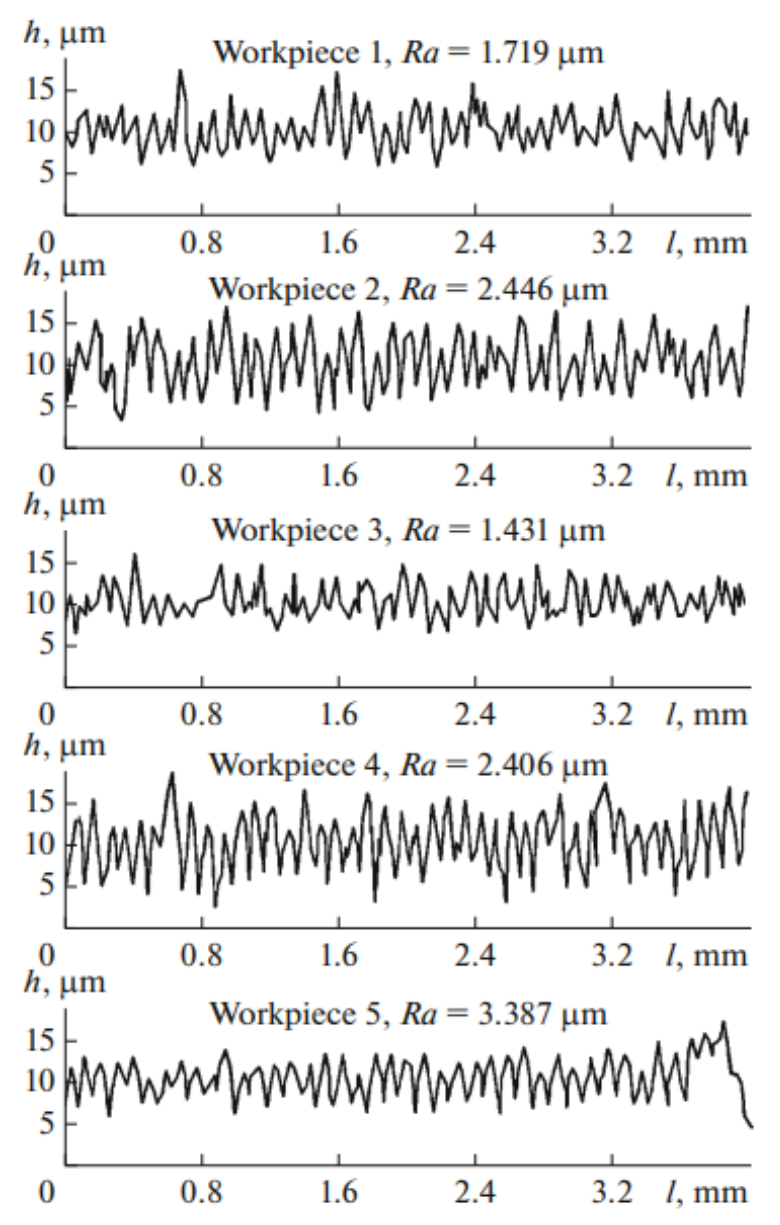

Fig. 1. Longitudinal profile-grams of rough surfaces of blanks made of steel 45. Horizontal increase x20; vertical increase $\mathrm{x} 1000$.

The longitudinal profile-gram of the calibrationstrip in the deforming drawplate [10] for determining the radius $R_{S W}$, is shown in Figure 2. A diamond tool (radius $1.5 \mathrm{~mm}$ ) is used to produce regular micro-relief, in the form of single-turn helical channels (groove depth: $D_{c h}=$ $10 \mu \mathrm{m}$, groove pitch: $P_{c h}=0.5 \mathrm{~mm}$ ). Here $R a_{t}$ is the surface roughness parameter of the working channel of the drawplate according to the calibrating strip (tool).

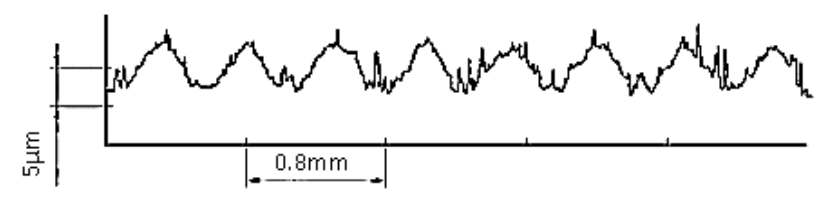

Fig. 2. Longitudinal profile-gram of the calibrating band for deforming the drawplate with a regular micro-relief: material 9XC, $R a_{t}=1.791 \mu \mathrm{m}$. Horizontal increase $\mathrm{x} 20$; vertical increase $\mathrm{x} 1000$ 
The drawing speed is $1 \mathrm{~m} / \mathrm{min}$. The lubricant employed is $I-40$ mineral oil with an admixture of oil-soluble "Valen" metal-coating additive at 50 vol \%, realizing the fundamental scientific principle "Garkunov-Kragelsky effect, friction without wear (selective transfer)" $[4,9,10,13$, 14, 25]. Figure 3 presents the schematic view of the experimental setup: a thin-walled cylinder 1 with a cavity 2 for lubricant. In the upper part of cylinder 1 , there is a machining drawplate 3 , whose working surfaces are hardened by a regular micro-relief (fragment $A$ ). When blank 4 passes through drawplate 3 into cavity 2 , the lubricant pressure attains the required value for flowing in the micro-relief channels on the working surface of drawplate 3 . In figure 3 fragment $A$ shows the parameter of the deformation zone $\left(\Delta R_{b}\right)$ in the form of a sign and wave height of non-contact deformation arising on the working cone of the drawplate, whereas fragment $\mathrm{B}$ shows the deformation zone.
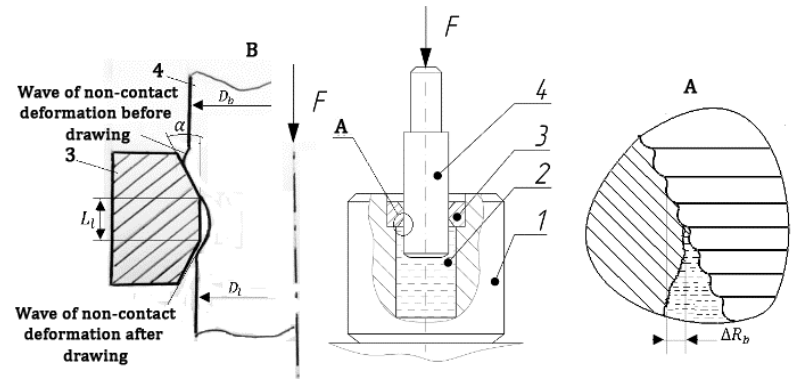

Fig. 3. The experimental setup, a) The parameter $\Delta R_{b}$, b) The deformation zone, 1- The thin-walled cylinder, 2- Cavity with lubricant, 3- Drawplate, 4- Workpiece.
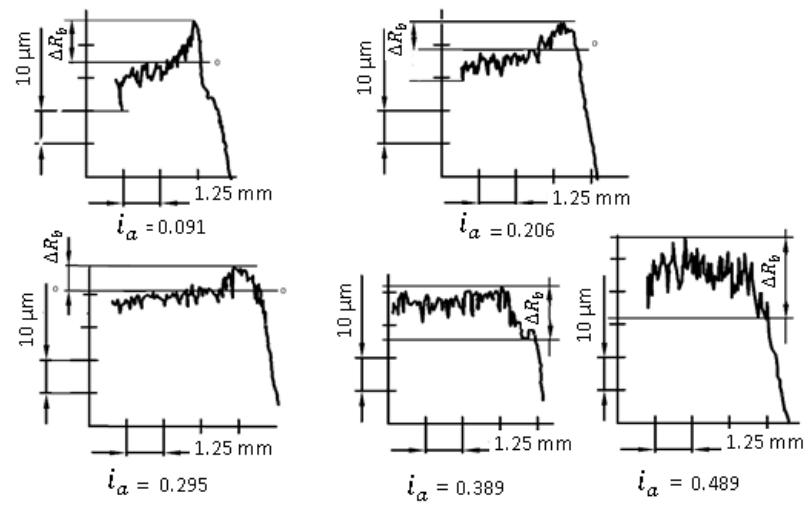

Fig. 4. Longitudinal profile-gram of the deformation zones at the working cone of the deforming drawplate: $D_{c h}=10 \mu \mathrm{m}, P_{c h}=0.5 \mathrm{~mm}$; steel 45; lubricant "I-40" $+50 \%$ of metal-clad additives; horizontal increase $x 8$; vertical increase $\mathrm{x} 1000$.
In order to determine $\Delta R_{b}$ (figure 3 fragment A), the profile-gram presented in figure 4 is considered. The analysis of figure 4 shows that a deformation zone is formed on the working cone of the drawplate in the form of a positive wave of non-contact deformation (fragment B) when $i_{a}<0.389 \mathrm{~mm}$ and in the form of a negative wave when $i_{a}>0.389 \mathrm{~mm}$. The results obtained by determining the parameter $\Delta R_{b}$ are presented in figure 5 .

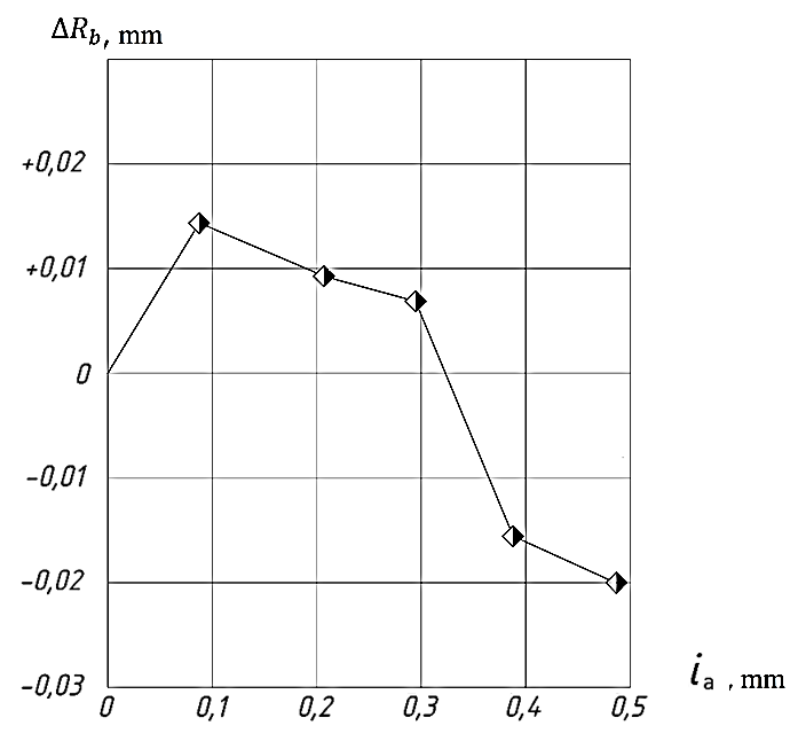

Fig. 5. The dependence of the magnitude and sign of non-contact deformation on the actual absolute deformation. $D_{c h}=10 \mu \mathrm{m}, P_{c h}=0.5 \mathrm{~mm}$; steel 45; lubricant "I-40" + 50\% metal-plating additives "Valen".

For exact calculations with the mathematical model (1), the hardening of the material used is tested according to GOST 25.503-97. The resulting hardening curve for steel 45 is shown in Figure 6. Where $e_{i}$ is the accumulated deformation of the workpiece (the degree of deformation) that is calculated by using expression (2):

$$
e_{i}=2 \ln \left(\frac{D_{b}+2 \Delta R_{b}}{D_{l}}\right)
$$

The accumulated deformation is a quantity whose total derivative in time is equal to the intensity of the deformation speed $\varphi_{i}$ (3):

$$
\varphi_{i}=\frac{d e_{i}}{d t}
$$


The resulting overall hardening curve for steel 45 is shown in Figure 6a. The considered values of the accumulated deformation of the workpiece $e_{i}$ [19] in the experimental study are in the range $e_{i}=0,011-0,044$; therefore, to obtain the equation of the hardening curve, the results are approximated in the range $e_{i}=0,00675-$ 0,045 . The partial hardening curve approximated in this interval $\left(e_{i}=0,00675-0,045\right)$ is shown in Figure $6 \mathrm{~b}$, and the expression for this hardening curve is:

$$
\sigma_{s}=241.061+5872 e_{i}
$$

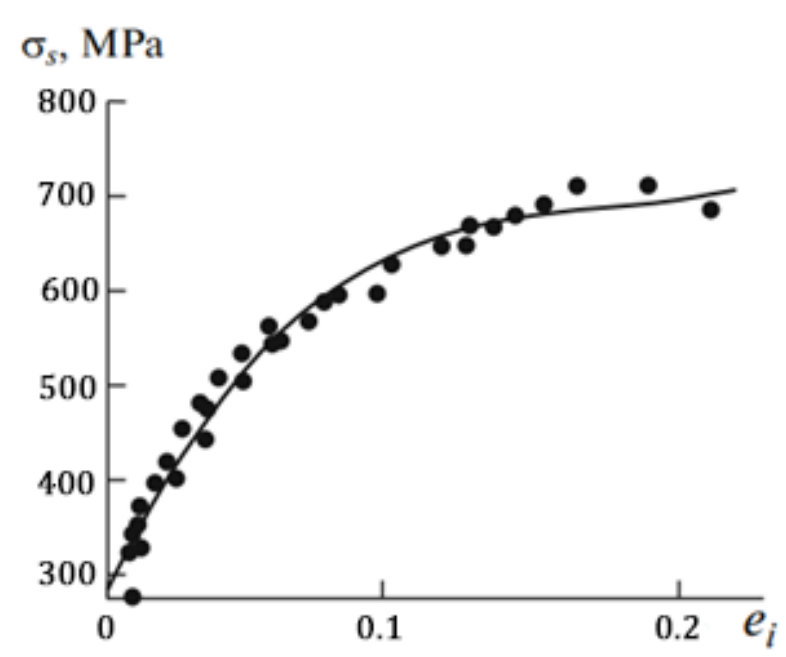

(a)

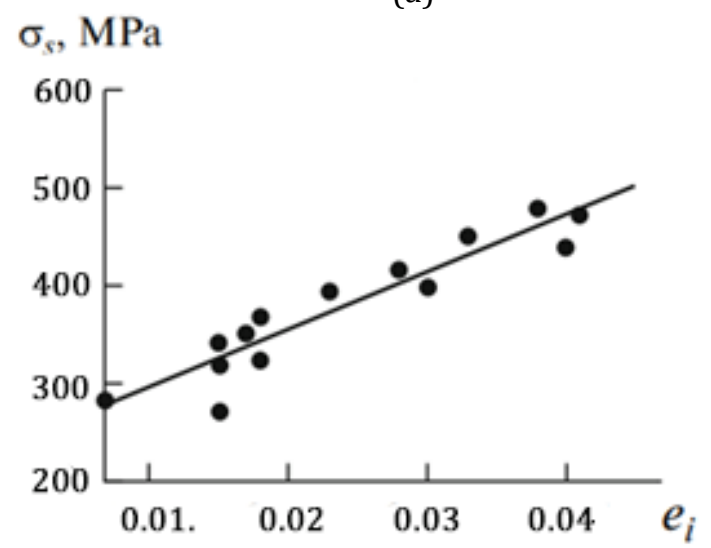

(b)

Fig. 6. Hardening curve of steel 45: a) total; b) partial

The initial information and the calculation results by the mathematical model, as well as the comparison of theoretical $q_{d}^{\mathrm{T}}$ and experimental $q_{d}^{e}$ specific drawing forces, are given in Table 1. The experimental drawing (broaching) specific force is determined from the expression $(\mathrm{N} /$ $\mathrm{mm})$ :

$$
q_{d}^{e}=49.363+364.31 i_{a}
$$

After the approximation of the results, the analytical dependence of the theoretical drawing (broaching) specific force is determined from the expression $(\mathrm{N} / \mathrm{mm})$ :

$$
q_{d}^{\mathrm{T}}=76.279+236.34 i_{a}
$$

Figure 7 represents a graphical interpretation of the comparison of both theoretical and experimental dependence of the drawing.

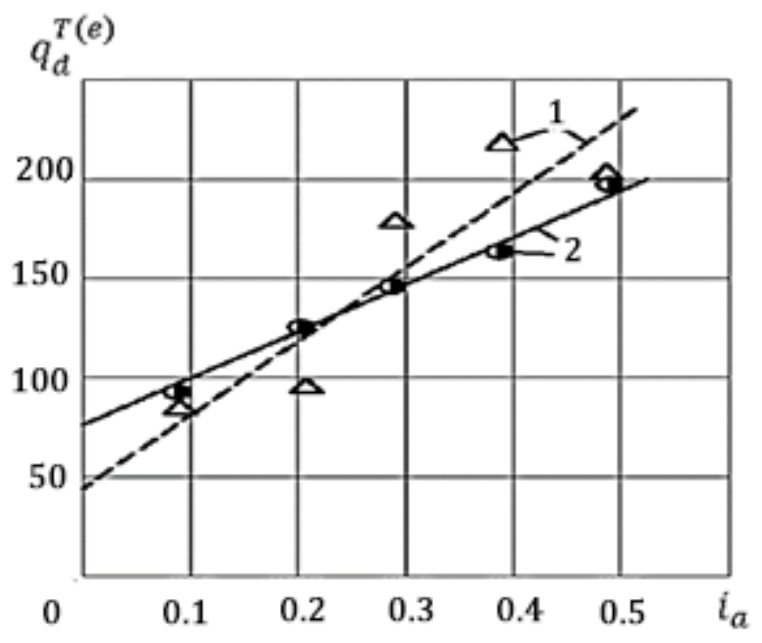

Fig. 7. Dependence of the specific drawing force on the absolute actual deformation: 1) experimental; 2) theoretical.

To estimate the theoretical contact pressure $q_{a}^{\mathrm{T}}$ on the working cone of the deforming element, the following dependencies are used:

The contact width $L_{a}$ of the working cone of the tool with the workpiece "hole"

$$
L_{a}=\frac{0.5 i_{a}+\Delta R_{b}}{\sin \alpha}
$$

The diameter $D_{c}$ of the working cone of the tool in the middle of the contact width between the tool and the workpiece "hole".

$$
D_{c}=0.5\left(2 D_{l}-\left(i_{a}+2 \Delta R_{b}\right)\right.
$$

According to the data in Table 1, the theoretical actual contact pressure $q_{a}^{\mathrm{T}}$ has a maximum value when $i_{a} \geq 0.389$.

This regularity is explained as follows: as the degree of deformation increases, the actual contact width grows faster than the theoretical total drawing force $F_{d}^{\mathrm{T}}$ and vice versa. 
Table 1. Baseline data for the calculation and comparison of the specific drawing force

\begin{tabular}{|c|c|c|c|c|c|}
\hline \multirow{2}{*}{ Parameter } & \multicolumn{5}{|c|}{ Workpiece } \\
\hline & 1 & 2 & 3 & 4 & 5 \\
\hline$i_{a}, \mathrm{~mm}$ & 0.091 & 0.206 & 0.295 & 0.389 & 0.489 \\
\hline$D_{b}, \mathrm{~mm}$ & 20.091 & 20.206 & 20.295 & 20.389 & 20.489 \\
\hline$\Delta R_{b}, \mathrm{~mm}$ & +0.014 & +0.009 & +0.007 & -0.016 & -0.020 \\
\hline$\chi_{b}$ & 1.367 & 0.345 & 1.132 & 1.340 & 1.441 \\
\hline$h_{s w}, \mu \mathrm{m}$ & 1.5 & 1.5 & 1.5 & 1.5 & 1.5 \\
\hline$e_{i}$ & 0.01186 & 0.02227 & 0.03066 & 0.03538 & 0.0444 \\
\hline $\bar{\sigma}_{s}, \mathrm{MPa}$ & 275.89 & 306.46 & 331.089 & 344.93 & 371.4 \\
\hline$D_{l}, \mathrm{~mm}$ & 20 & 20 & 20 & 20 & 20 \\
\hline$\alpha, \operatorname{grad}$ & 5 & 5 & 5 & 5 & 5 \\
\hline$L_{l}, \mathrm{~mm}$ & 5 & 5 & 5 & 5 & 5 \\
\hline$H_{b \max }, \mu \mathrm{m}$ & 11.5 & 13 & 9.0 & 15.5 & 21.0 \\
\hline$\varepsilon_{b}$ & 0.896 & 0.920 & 0.917 & 0.940 & 0.953 \\
\hline$R_{c S}, \mu \mathrm{m}$ & 8727 & 8727 & 8727 & 8727 & 8727 \\
\hline$K_{1}$ & 3.778 & 3.778 & 3.778 & 3.778 & 3.778 \\
\hline$K_{2}$ & 0.408 & 0.408 & 0.408 & 0.408 & 0.408 \\
\hline$K_{3}$ & 1.220 & 1.220 & 1.220 & 1.220 & 1.220 \\
\hline$\tau_{0}, \mathrm{MPa}$ & 9.873 & 9.873 & 9.873 & 9.873 & 9.873 \\
\hline$\beta$ & 0 & 0 & 0 & 0 & 0 \\
\hline$f$ & 0.016 & 0.017 & 0.016 & 0.016 & 0.017 \\
\hline$q_{d}^{e}, \mathrm{~N} / \mathrm{mm}$ & 85.8 & 95.96 & 178.37 & 217.89 & 204.34 \\
\hline$q_{d}^{\mathrm{T}}, \mathrm{N} / \mathrm{mm}$ & 93.65 & 123.9 & 148.93 & 167.7 & 198.82 \\
\hline$\Delta q_{d}, \%$ & -9.14 & -29.1 & +16.5 & +23 & +2.7 \\
\hline$q_{a}^{\mathrm{T}}, \mathrm{MPa}$ & 1595 & 1517.7 & 1400.8 & 1893.3 & 2235 \\
\hline
\end{tabular}

\subsection{Second experimental study}

The second experimental basis, intended for the verification of the reliability of the theoretical model of the drawing force (1), adopts a basic approach similar to the one implemented in the first experimental basis. However, the second experience slightly differs from the first one in terms of the samples used which represent solid cylindrical billets made of steel 45 (186 HB) with a complicatedly modified surface layer; including a brass layer, a regular microgeometry (Fig. 8) and a "servowitte" copper layer [12]. These modifications resulted in a wave absence in the deformation zone (Fig. 9) [11, 15]. Tests were carried out on five specimens that have the following diameters: $D_{b}=20.133 \mathrm{~mm}, D_{b}=20.246 \mathrm{~mm}, D_{b}$ $=20.335 \mathrm{~mm}, D_{b}=20.453 \mathrm{~mm}$ and $D_{b}=20.560$ $\mathrm{mm}$. The actual absolute deformation is calculated by the expression $i_{a}=D_{b}-D_{l}$; where $D_{l}=20 \mathrm{~mm}$ is the diameter over the calibration bland (tool diameter figure 3). Therefore, the first specimen is deformed by the actual absolute deformation $i_{a}=0.133 \mathrm{~mm}$, the second by $i_{a}=0.246 \mathrm{~mm}$, the third by $i_{a}=0.335 \mathrm{~mm}$, the fourth by $i_{a}=0.453 \mathrm{~mm}$, and the fifth by $i_{a}=0.56$ $\mathrm{mm}$. The length of the drawn workpiece is 150 $\mathrm{mm}[10,13]$.

The initial data for the calculation and comparison of the theoretical specific drawing force are given in Table 2.

In this case, taking into account the preliminary plastic deformation and the regularization of the microgeometry of the surface of the samples (the depth of the grooves of $20 \mu \mathrm{m}$ ) [11, 12, 15] (Fig. $8,9)$ the hardening curve takes the following expression (MPa):

$$
\sigma_{s}=264+5872 e_{i}
$$




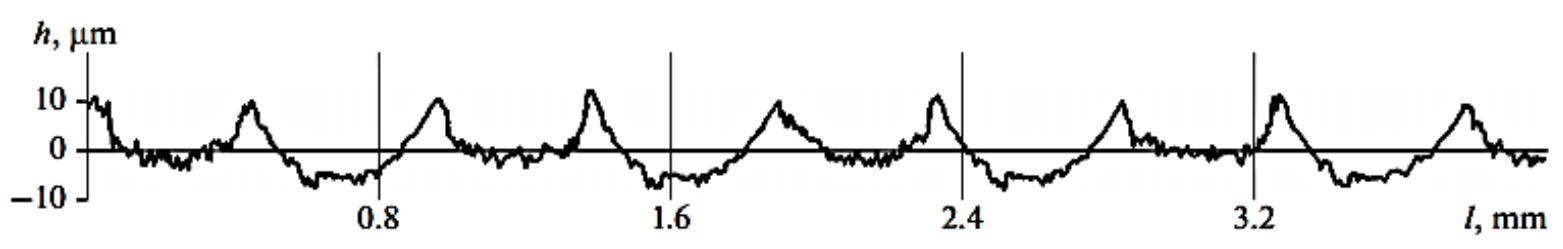

Fig. 8. Longitudinal profilogram of the surface of a cylindrical billet of steel 45 .

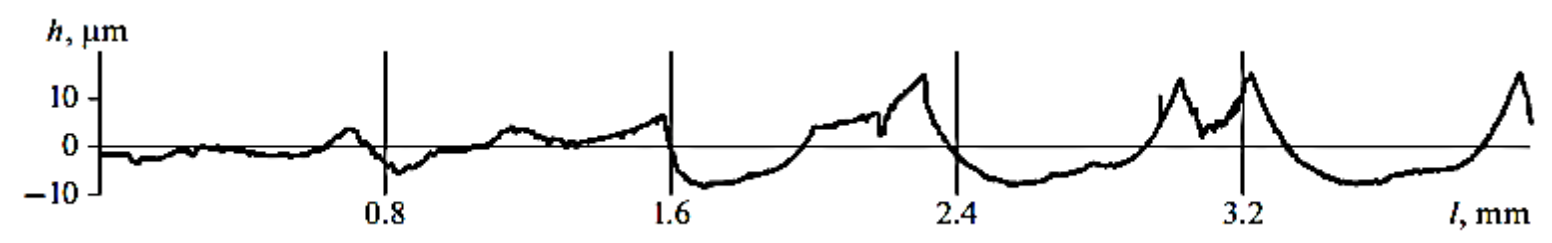

Fig. 9. The longitudinal profilogram of the deformation zone arising from the working cone of the deforming draw plate.

Therefore, the coefficient of sliding friction is determined by the expression [5]:

$$
f=f_{a}+f_{d}=\left(\frac{\tau_{0}}{H B_{b}}+\beta\right)+\left(0.28 \sqrt{\frac{\varepsilon_{b} H_{\text {bmax }}}{R_{s w}}}\right)
$$

Where $H B_{b}$ - surface hardness, MPa;

$R_{s w}$ - The radius of curvature at the summit of a single micro protrusion of the rough surface of the working channel in the deforming drawplate, $\mu \mathrm{m}$;

The reduced radius of curvature at the summit of single micro-protrusions of the rough surface $R_{s w}$ is obtained by the expression (11) given in [26].

$$
R_{s w}=\frac{4}{\frac{1}{R_{b_{\mathrm{x}}}}+\frac{1}{R_{b_{y}}}+\frac{1}{R_{t_{\mathrm{x}}}}-\frac{1}{R_{t_{\mathrm{y}}}}}
$$

Where $R_{b_{\mathrm{x}}}, R_{b_{y}}, R_{t_{\mathrm{x}}}, R_{t_{\mathrm{y}}}$ - respectively represent, the longitudinal $(\mathrm{x})$ and transverse $(\mathrm{y})$ radius at the summit of single micro-protrusions of the rough surfaces of workpiece (b) as well as the working channel of the deforming drawplate $(t)$ in $\mu \mathrm{m}$, [23].

After approximation of the experimental and the theoretical specific force values, the analytical dependencies are obtained as follows: $(\mathrm{N} / \mathrm{mm})$ :

$$
q_{d}^{e}=214.826+290.843 i_{a}
$$

$$
q_{d}^{\mathrm{T}}=67.11+533.65 i_{a}
$$

The analysis of expressions (12) and (13) show that they converge at $i_{a}=0,608 \mathrm{~mm}$. Figure 10 represents a graphical interpretation of the comparison of both the theoretical (12) and the experimental (13) dependences of the specific drawing (broaching) force.

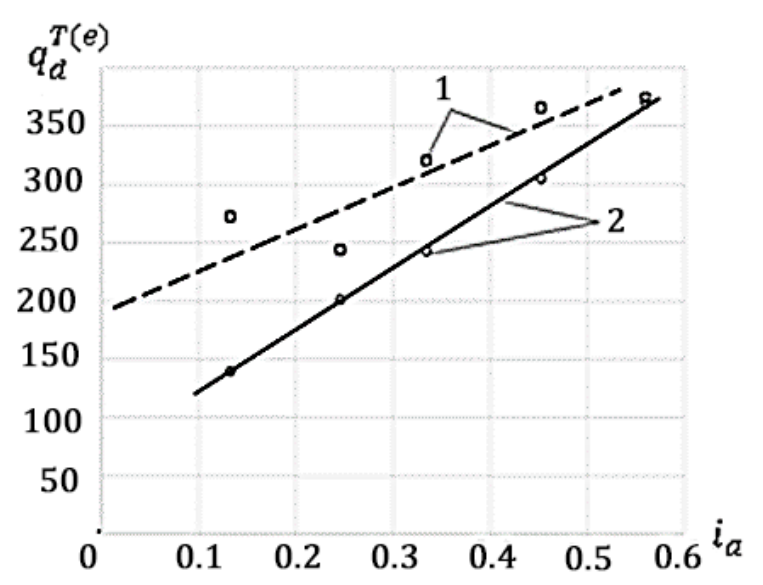

Fig. 10. Dependence of the specific drawing force on the absolute actual absolute deformation: 1) Experimental; 2) Theoretical.

To conclude, we notice that in the deformation zone, the wave in non-contact deformations is missing. This leads automatically to the absence of the actual contact width of the working cone of the deformed element with the workpieces. Thus, the contact pressure is practically missing on the working cone. 
Table 2. Baseline data for the calculation and comparison of the theoretical specific drawing force of solid cylindrical billets with a complexly modified surface layer

\begin{tabular}{|c|c|c|c|c|c|}
\hline \multirow{2}{*}{ Parameters } & \multicolumn{5}{|c|}{ Workpieces } \\
\cline { 2 - 6 } & 1 & 2 & 3 & 4 & 5 \\
\hline$i_{a}, \mathrm{~mm}$ & 0.133 & 0.246 & 0.335 & 0.453 & 0.56 \\
\hline$D_{b}, \mathrm{~mm}$ & 20.133 & 20.246 & 20.335 & 20.453 & 20.560 \\
\hline$H_{b \text { max }}, \mu \mathrm{m}$ & 28.37 & 28.833 & 28.833 & 28.832 & 28.82 \\
\hline$\varepsilon_{b}$ & 0.5169 & 0.963 & 0.959 & 0.9518 & 0.95 \\
\hline$e_{i}$ & 0.01325 & 0.0244 & 0.0332 & 0.04479 & 0.0552 \\
\hline $\bar{\sigma}_{s}, \mathrm{MPa}$ & 291.64 & 324.5 & 350.2 & 384.09 & 414.66 \\
\hline$\Delta R_{b}, \mathrm{~mm}$ & 0 & 0 & 0 & 0 & 0 \\
\hline$B$ & 0 & 0 & 0 & 0 & 0 \\
\hline$\tau_{0}, M P a$ & 42.965 & 42.965 & 42.965 & 42.965 & 42.965 \\
\hline$H B_{b}, M P a$ & 1860 & 1860 & 1860 & 1860 & 1860 \\
\hline$R_{t_{X}}, \mu \mathrm{m}$ & 3125 & 3125 & 3125 & 3125 & 3125 \\
\hline$R_{t_{y}}, \mu \mathrm{m}$ & 10000 & 10000 & 10000 & 10000 & 10000 \\
\hline$R_{b_{X}}, \mu \mathrm{m}$ & 273 & 273 & 273 & 273 & 273 \\
\hline$R_{b_{y}}, \mu \mathrm{m}$ & 10065 & 10123 & 10167 & 10226 & 10280 \\
\hline$R_{c s}, \mu \mathrm{m}$ & 1007 & 1007 & 1007 & 1007 & 1007 \\
\hline$f_{a}$ & 0.023 & 0.023 & 0.023 & 0.023 & 0.023 \\
\hline$f_{d}$ & 0.03378 & 0.04649 & 0.04639 & 0.04622 & 0.0461 \\
\hline$F$ & 0.05678 & 0.06949 & 0.06939 & 0.0692 & 0.0691 \\
\hline$D_{l}, \mathrm{~mm}$ & 20 & 20 & 20 & 20 & 20 \\
\hline$\alpha, \mathrm{grad}$ & 5 & 5 & 5 & 5 & 5 \\
\hline$L_{l}, \mathrm{~mm}$ & 5 & 5 & 5 & 5 & 5 \\
\hline$q_{d}^{\mathrm{T}}, \mathrm{N} / \mathrm{mm}$ & 139.156 & 200.44 & 243.24 & 305.38 & 369 \\
\hline$q_{d}^{e}, \mathrm{~N} / \mathrm{mm}$ & 272.29 & 243.63 & 320.06 & 366.24 & 374.2 \\
\hline$\Delta q_{d}, \%$ & +48.9 & +17.7 & +24 & +16.6 & +1.38 \\
\hline & & & & & \\
\hline
\end{tabular}

\section{DISCUSSION}

The literature review reveals that the modules of predicting the drawing force have shown relative errors in percentage $\left(\Delta q_{d}=\left(\frac{q_{d}^{e}-q_{d}^{\mathrm{T}}}{q_{d}^{e}}\right) 100 \%\right)$ which are largely different regarding the theoretical and the practical results. In general, previous studies have shown that the relative error in percentage between theoretical and experimental data ranges from 20 to $200 \%$. The smallest relative percentage errors referred to as $\Delta q_{d}$ are given through the solutions proposed by A. A. Dinnik (error 5-20\%), M.M. Bernstein (7$30 \%)$, and A.L. Vorontsov (2-12\%). The modules we have proposed give the following results: for steel 45 samples without surface modification, the relative percentage errors range from -29.1 to $+23 \%$, and for steel 45 samples with surface modification, the relative percentage errors range from +1.38 to $+48.9 \%$.

For in-depth analysis, we will compare the results obtained in the first and second case with the results of Vorontsov (table 3). In order for the comparison to be accurate, we will stick to the case where Vorontsov used the brass samples and the tool with an angle " $\alpha=5$ grad" and "width of the calibrating ribbon of tool $l=0$ ".

For the modules proposed in the first case, the relative percentage error ranges from -16.45 to 12.441 if $i_{a}$ is between 0.1 and 0.4 . The relative percentage of error is acceptable for the practice of engineering. In the second case, if $i_{a}$ is between 0.45 and 0.9 , the relative percentage of error ranges from -14.85 to 11,123 . Also, this is acceptable in engineering practice. 
The main advantage of the current proposed modules is the ability to calculate the values of $q_{d}^{\mathrm{T}}$ and $q_{d}^{e}$ regardless of the value of $i_{a}$; the thing which was impossible to achieve with the previous proposed modules.

The performance of the predictive models was measured using root mean -square error (RMSE)

$$
R M S E=\sqrt{\frac{\sum_{1}^{n}\left(q_{d}^{T}-q_{d}^{e}\right)^{2}}{n}}
$$

The modules proposed in the first case give that the $R M S E=6.6205$ when $i_{a}$ ranges from 0.15 to 0.3 and the $R M S E=29.183$ when $i_{a}$ ranges from 0.091 to 0.489 , and $R M S E=0$ when $i_{a}=0.210$. In the second case, the modules proposed give that the $R M S E=17.2888$ when $i_{a}$ ranges from 0.5 to 0.8 and the $R M S E=76.4491$ when $i_{a}$ ranges from 0.133 to 0.56 , and $R M S E=0$ when $i_{a}=$ 0.608 .

Table 3. The results obtained by the method of Vorontsov and by the proposed modules.

\begin{tabular}{|c|c|c|c|c|c|}
\hline \multirow{2}{*}{ Vorontsov's Module } & \multicolumn{4}{|c|}{ Proposed Modules } \\
\cline { 3 - 6 } & & \multicolumn{2}{|c|}{ first case } & \multicolumn{2}{c|}{ Second case } \\
\hline$i_{a}$ & $\Delta q_{d} \%$ & $i_{a}$ & $\Delta q_{d} \%$ & $i_{a}$ & $\Delta q_{d} \%$ \\
\hline 0.5 & 0.8 & 0.15 & -7.42 & 0.55 & 3.78 \\
\hline 0.8 & 1.3 & 0.2 & -1.08 & 0.6 & 0.52 \\
\hline 1.7 & 3.7 & 0.215 & 0.46 & 0.7 & -5.31 \\
\hline 1.5 & 5 & 0.25 & 3.61 & 0.8 & -10.39 \\
\hline
\end{tabular}

The analysis of the results (Table 1) shows that in the range $i_{a}=0,091-0,489 \mathrm{~mm}$, the relative percentage error of the theoretical values of the drawing force ranges from - 29.1 to $+23 \%$; whereas, the experimental dependence (5) and the theoretical dependence (6) converge when $i_{a}=$ $0,21 \mathrm{~mm}$ (Fig. 7). The possible reasons for the occurrence of large errors between the prediction and experiment are the longitudinal vibration of the fixture and the widespread roughness parameters of the surface of the workpieces before drawing (Fig. 1, table 1); which eventually affects the mechanisms involved significantly in a tribological contact in accordance with the applied formulas of the coefficient of sliding friction $f=f_{a}+f_{d}$ in expression (1) and the actual contact pressure $q_{a}^{\mathrm{T}}$.

This actual contact pressure exceeds the average yield stress of the processed material $\left(q_{a}^{\mathrm{T}} \gg \bar{\sigma}_{s}\right)$ significantly over the deformation zone. This indicates a similar ratio of the actual and nominal contact area between the workpiece (rough surfaces) and the working channel of the deforming drawplate (Fig. 1, 2) $[4,5]$. The Contact pressure exceeds the average yield stress that leads to the stimulation of the stresses in the workpiece, resulting in deep micro-cracks that, in their turn, pass into the finished product's surface layer and reduce its performance.
The use of metal lubricants, tools and workpieces with a regular micro-relief has been proven to be very efficient. This has allowed a significant reduction in friction and deformation energy costs as well as a contribution to a complete elimination of the adhesion of the processed material.

While using the samples with an irregular microrelief (Fig. 1), a positive wave of non-contact deformation arises in the drawplate's working cone, which increases the contact area between the tool surface and the workpiece surface, and then making it difficult for the lubricant to get into the deformation zone. As a consequence, the realization of the hydrodynamic lubrication regime is disrupted, leading to an increase in the processing force.

The wave of non-contact deformation causes the tensile stresses and the formation of cracks passing onto the surface layer. This leads to low product life, poor metallurgical properties, and overall poor product quality.

When using the samples with a regular microrelief (Fig. 8), the material, being thrust by the drawplate, fills in each groove of the regular micro-relief, causing a complete absence of the non-contact deformation negative/positive wave on the active cone of the tool (working cone of draw plate) (fig. 9) $[11,15]$. 
The effect of "the regular micro-relief grooves" on the specimen surface may be explained by the violation of the deformable layer continuity.

For future studies, the proposed modules can be applied to the tools with the optimal geometric parameters determined by Vorontsov. The optimal geometric parameters are the angles $\alpha,(\alpha=6, \alpha=$ 8, $\alpha=12, \alpha=20, \alpha=21.5$ ) and the width of the calibrating ribbon $L_{l}\left(L_{l}=0, L_{l}=1, L_{l}=2, L_{l}=7\right)$.

\section{CONCLUSION}

Based on the review of literature and the experiments that have been carried out, some conclusions are deduced:

- A mathematical module to predict the drawing forces of continuous cylindrical parts is formed and verified. The modification is made to take into account the geometrical parameters of the deformation zone and the "selective transfer phenomenon". For the verification, two identical experiments are conducted. The first experiment, the samples are machined by turning. The second experiment, the specimens are distinguished by the superficial layer, which is modified, by a brass-coated layer, a regular microgeometry and a copper layer. As a result, in the first experiment, the differences between theoretical and experimental force values are in the range of $-29.1 \%$ to $+23 \%$ whereas in the second experiment, the differences are in the range of $+1.38 \%$ to $+48.9 \%$.

- According to the experimental and analytical results, the maximum force ( $q_{d}^{\mathrm{T}}=369 \mathrm{~N} / \mathrm{mm}$ and $q_{d}^{e}=374.2 \mathrm{~N} / \mathrm{mm}$ with $\Delta q_{d}=+1.38 \%$ ) is obtained when there is no wave of non-contact in the deformation zone and at $i_{a}=0.56 \mathrm{~mm}$.

- The regular micro-relief has a positive effect on the utilization of both the final part and the machining of the blank one.

- The regular micro-relief also increases the precision of the part in terms of noncircular deformation and distortion of the longitudinal cross-section.

Furthermore, the results will expand the database for systematic parametric synthesis of new hybrid machining methods. Overall, this research confirms that various combined machining methods may be fundamentally improved by employing Garkunov-Kragelsky wear - free conditions.

\section{REFERENCES}

[1] P. Mahesh, S. Varinder, S.P. Regalla, A.K. Gupta, T.C. Bera, B. Simhachalam, K. Srinivasa, Parametric optimization of the generation of the porous layer for lubrication in tube drawing process, Materials Today: Proceedings, vol. 28. part 3, 2020, doi: 10.1016/j.matpr.2020.04.840

[2] U.S. Dixit, R.G. Narayanan, Metal Forming: Technology and Process Modelling, Tata McGraw Hill, 2013.

[3] T. Altan, H. Gegel, Metal Forming: Fundamentals and Applications, American Society for Metals, 2000.

[4] M. Nosonovsky, V. Mortazavi, Friction-Induced Vibrations and Self-Organization: Mechanics and Non-Equilibrium Thermodynamics of Sliding Contact, CRC Press, 2013, doi: 10.1201/b15470-9

[5] A.V. Shchedrin, V.V. Ulyanov, A.A. Bekaev, V.M. Skoromnov, M.S. Vanyushkina, T.G. Abramova, N.V. Khomyakova, N.Yu. Chikhacheva, Tribological Principles in the Systematic Design of Competitive Technological Equipment, Repair, restoration, modernization, no. 4 , pp. 15-20, 2009. (in Russian)

[6] Y.G. Kabaldin, D.A. Shatagin, Artificial Intelligence and Cyberphysical Machining Systems in Digital Production, Russian Engineering Research, vol. 40, pp. 292-296, 2020, doi: 10.3103/s1068798x20040115

[7] W. Hu, L.G. Yao, Z.Z. Hua, Optimization of sheet metal forming processes by adaptive response surface based on intelligent sampling method, Journal of Materials Processing Technology, vol. 197, iss. 1-3, pp. 77-88, 2008, doi: 10.1016/j.jmatprotec.2007.06.018

[8] A.V. Shchedrin, V.V. Ul'yanov, V.M. Skoromnov, A.A. Bekaev, Benefits of a draw plate with regular microrelief, Russian Engineering Research, vol. 28, pp. 357-361, 2008, doi: 10.3103/S1068798X08040163

[9] S.A. Gavrilov, A.V. Shchedrin, Improved Use of MetalPlating Conditioner in Combined Machining, Russian Engineering Research, vol 32. iss. 7-8, pp. 573-576, 2012, doi: $10.3103 / \mathrm{S} 1068798 X 1206007 \mathrm{X}$

[10] Y. Zhang, R.R. Chromik, Tribology of SelfLubricating Metal Matrix Composites, in P.L. 
Menezes, P.K. Rohatgi, E. Omrani, (Eds.): SelfLubricating Composites, Springer, pp. 3373, 2018, doi: 10.1007/978-3-662-56528-5_2

[11] A.V. Shchedrin, A.Yu. Kozlov, A.A. Kostryukov, Improvement of the covering surface plastic deformation due to the regularization of microgeometry of the surface of the workpiece, Strengthening technologies and coatings, vol. 13, no. 4, pp. 162-168, 2017. (in Russian)

[12] A.V. Shchedrin, A.A. Kostryukov, Application of tribotechnology based on self-organization for the systematic improvement of the processes of cold plastic deformation, Strengthening technologies and coatings, vol. 13, no. 11, pp. 495-499, 2017. (in Russian)

[13] A.A. Kostryukov, A.V. Shchedrin, V.F. Aleshin, K.O. Klimochkin, V.B. Esov, Improvement of impact method on processed material in covering superfi cial plastic deformation methods, Blanking productions in mechanical engineering, vol. 15, no. 5, pp. 211-216, 2017. (in Russian)

[14] P.D. Srivyasa, M.S. Charoo, Effect of Sintering Temperature and Reinforcement Concentration on the Tribological Behaviors of Hybrid Aluminum Matrix Nano Composite, Tribology in Industry, vol. 41, no. 4, pp. 573-591, 2019, doi: 10.24874/ti.2019.41.04.10

[15] A.V. Shchedrin, A.A. Kostryukov, E.L. Mel'nikov, V.Yu. Lavrinenko, V.F. Aleshin, N.Yu. Chikhacheva, Perfection of covering surface plastic deformation of billets from aluminum alloys, Strengthening technologies and coatings, vol. 14, no. 7, pp. 291-296, 2018. (in Russian)

[16] E.L. Melnikov, A.V. Shchedrin, A.A. Kostryukov, M.A. Seryozhkin, V.P. Stupnikov, V.V. Stupnikov, Method of cold plastic deformation of metals, Patent no. RU2647057C1, 2017. (in Russian)

[17] A. A. Udalov, A.V. Udalov, S.V. Parshin, Influence of Deformation Conditions on the Power Regimes of the Process of Cold Crimping of a Pipe Billet in a Conical Die, Materials Science Forum, vol. 946, pp. 812-817, 2019, doi: 10.4028/www.scientific.net/msf.946.812
[18] H. Kim, K.S. Cho, B.C. Ji, Mathematical modelling of zone drawing process, Macromolecular Research, vol. 12, pp. 206-212.,2019, doi: $10.1007 / \mathrm{bf03218389}$

[19] V.Ya. Osadchy, A.L. Vorontsov, Formula to calculating the drawing stress of round solid profiles, Proizvodstvo Prokata, no. 6, pp. 3-8, 2001. (in Russian)

[20] A.V. Grushko, V.V. Kukhar, Y.O. Slobodyanyuk, Phenomenological Model of Low-Carbon Steels Hardening during Multistage Drawing, Solid State Phenomena, vol. 265, pp. 114-123, 2017, doi: 10.4028/www.scientific.net/ssp.265.114

[21] J. Cao, E. Brinksmeier, M. Fu, R. X. Gao, B. Liang, M. Merklein, J. Yanagimoto, Manufacturing of advanced smart tooling for metal forming, CIRP Annals, vol. 68, iss. 2, pp. 605-628, 2019, doi: 10.1016/j.cirp.2019.05.001

[22] A.V. Shchedrin, S.A. Gavrilov, I.V. Kosarev, T.V. Smolkina, M.A. Zinin, E.S. Sergeev, Formation of foci of deformation in the surrounding surface plastic deformation, Friction and lubrication in machines and mechanisms, no. 10, pp. 3-7, 2014. (in Russian)

[23] V.A. Kuznetsov, A.V. Schedrin, S.A. Gavrilov, V.I. Voronkov, E.L. Mel'nikov, Theoretical and experimental researches of encompassing superficial plastic deformation method in conditions of using of metal-cladding lubricants, Strengthening technologies and coatings, no. 2, pp. 11-17, 2014. (in Russian)

[24] V.G. Babel, D.N. Garkunov, D.N. Garkunov, S.M. Mamykin, S.M. Mamykin, P.I. Kornik, P.I. Kornik, Metal-containing oil-soluble composition for lubricant materials, Patent no. RU2277579C1, 2005.

[25] N.S. Lidorenko, Scientific discovery, Association of the authors of scientific discoveries of the USSR, no. 41, Moscow, 1996. (in Russian)

[26] M.A. Chowdhury, Friction, Lubrication and Wear, London: IntechOpen, 2019 doi: 10.5772/intechopen.77584 